\title{
Depth of Field Affects Perceived Depth in Stereographs
}

\author{
TINGTING ZHANG, Delft University of Technology \\ LOUISE O'HARE, University of Lincoln \\ PAUL B. HIBBARD, University of Essex \\ HAROLD T. NEFS, Delft University of Technology \\ INGRID HEYNDERICKX, Eindhoven University of Technology
}

\begin{abstract}
Although it has been reported that depth of field influences depth perception in nonstereo photographs, it remains unclear how depth of field affects depth perception under stereo viewing conditions. We showed participants stereo photographs with different depths of field using a Wheatstone stereoscope and a commercially available 3D TV. The depicted scene contained a floor, a background, and a measuring probe at different locations. Participants drew a floor plan of the depicted scene to scale. We found that perceived depth decreased with decreasing depth of field for shallow depths of field in scenes containing a heightin-the-field cue. For larger depths of field, different effects were found depending on the display system and the viewing distance. There was no effect on perceived depth using the 3D TV, but perceived depth decreased with increasing depth of field using the Wheatstone stereoscope. However, in the 3D TV case, we found that the perceived depth decreased with increasing depth of field in scenes in which the height-in-the-field cue was removed. This indicates that the effect of depth of field on perceived depth may be influenced by other depth cues in the scene, such as height-in-the-field cues.
\end{abstract}

Categories and Subject Descriptors: I.4.8 [Scene Analysis]: Depth Cues; I.2.10 [Vision and Scene Understanding]: 3D/ Stereo Scene Analysis; I.3.1 [Hardware Architecture]: Three-Dimensional Displays

General Terms: Human Factors

Additional Key Words and Phrases: Depth of field, depth perception, stereoscope, 3D TV, binocular disparity

ACM Reference Format:

Tingting Zhang, Louise O'hare, Paul B. Hibbard, Harold T. Nefs, and Ingrid Heynderickx. 2014. Depth of field affects perceived depth in stereographs. ACM Trans. Appl. Percept. 11, 4, Article 18 (December 2014), 18 pages.

DOI: http://dx.doi.org/10.1145/2667227

\section{INTRODUCTION}

Depth of field is the depth range around the focal plane that is perceived as sharp. All optical lens systems, including the human eye and digital photo cameras, generate images with a limited depth of

Tingting Zhang was supported by a CSC scholarship from the Chinese government. Louise O'Hare was funded by a doctoral training grant from the BBSRC.

Authors' addresses: T. Zhang and H. T. Nefs, Mekelweg 4, HB 12.100, NL 2628CD Delft, email: t.zhang@tudelft.nl, h.t.nefs@ tudelft.nl; L. O'Hare, Brayford pool, Lincoln, Lincolnshire, LN6 7TS, United Kindom; email: lohare@lincoln.ac.uk; P. B. Hibbard, Room 3.716, Wivenhoe Park, Colchester, CO4 3SQ, United Kindom; email: phibbard@essex.ac.uk; I. Heynderickx, email: I.E.J. Heynderickx@tue.nl.

Permission to make digital or hard copies of part or all of this work for personal or classroom use is granted without fee provided that copies are not made or distributed for profit or commercial advantage and that copies show this notice on the first page or initial screen of a display along with the full citation. Copyrights for components of this work owned by others than ACM must be honored. Abstracting with credit is permitted. To copy otherwise, to republish, to post on servers, to redistribute to lists, or to use any component of this work in other works requires prior specific permission and/or a fee. Permissions may be requested from Publications Dept., ACM, Inc., 2 Penn Plaza, Suite 701, New York, NY 10121-0701 USA, fax +1 (212) 869-0481, or permissions@acm.org.

(C) 2014 ACM 1544-3558/2014/12-ART18 $\$ 15.00$

DOI: http://dx.doi.org/10.1145/2667227 
field, as long as they are focused nearer than the hyperfocal distance. Depth of field depends on the size of the lens aperture relative to the focal distance of the imaging devices. The amount of blur of an object in the scene depends on the distance of that object to the focal plane and can be expressed as

$$
b=A \frac{s_{0}}{d_{0}}\left|1-\frac{d_{0}}{d_{1}}\right|,
$$

where $b$ is the diameter of the blur circle (i.e., the diameter of the area on the projection plane over which a point is spread out), $A$ is the diameter of the lens aperture, $s_{0}$ is the distance from the lens to the image plane, $d_{0}$ is the distance from the lens to the focal plane, and $d_{1}$ is the distance from the focal plane to an object in the scene. A different form of this equation can be found in Nefs [2012]. Hence, depth of field can be considered as the distance range in which the blur circles remain smaller than the smallest amount of perceptible blur. Equation (1) shows that the blur circle $b$ at a certain depth distance $d_{1}$ increases as the focal distance $d_{0}$ decreases on the condition that the relative distance $d_{0} / d_{1}$ is fixed. In other words, when the focal distance decreases, depth of field becomes smaller as well. However, if the focal distance $d_{0}$ is fixed, the depth distance $d_{1}$ can be directly estimated from the blur circle based on Equation (1), assuming that $A, d_{0}$, and $s_{0}$ are known. When an optical system has a small depth of field, the blur circle $b$ will rapidly increase with increasing distance from the focal plane; thus, people will perceive a large blur gradient in the resulting images.

Depth of field is an important aspect of vision, and there has been significant research on the effects of depth of field in nonstereo photographs over the past few years. For example, it has been shown that depth of field can be used to direct viewers' attention [Baveye et al. 2012; Hamerly and Dvorak 1981; Hillaire et al. 2008; Khan et al. 2010], and it may also influence the aesthetic appreciation of images [Datta et al. 2006]. Other research has directly assessed its effects on the perceived distance from the observer to objects and the perceived distances between objects in the scene [Held et al. 2010; Mather 1996; Mather and Smith 2000; Pentland 1987; Vishwanath and Blaser 2010; Watt et al. 2005].

In the current study, we assess the effect of depth of field on the perception of depth in stereo photographs. A strong perception of depth can be created using stereoscopic presentation. Stereoscopic images and videos are widely used in many fields, such as cinematographic industries, virtual reality, visualization, and computer-assisted design [Hubona et al. 1999]. However, the challenge of delivering a high-quality, comfortable experience of depth perception using current display technology remains an active and important area of research [Banks et al. 2012; Shibata et al. 2011]. We investigated the extent to which depth of field can be used as a cue to depth complementing that provided by binocular disparity. To do this, we investigated the effects of depth of field on depth perception in stereoscopically presented photographs using two different presentation methods.

\subsection{Depth of Field in Nonstereo Photographs}

Previous research has already investigated the effect of depth of field on perceived depth in nonstereo photographs. Before considering the results of these experiments, it is important to appreciate the different ways in which depth of field may affect the perception of depth. In the first instance, let us assume that the viewing distance is known, and that the parameter $d_{0}$ in Equation (1) is therefore fixed and known. In this case, the depth of field could be calculated, and the amount of blur could be used as a cue to depth. Thus, a small blur circle would be interpreted as the result of viewing an object with a small deviation in distance from the focal plane (i.e., $d_{1}$ is similar to $d_{0}$ ). The consequence of decreasing the depth of field and increasing the amount of blur in the scene would in this case be to increase the magnitude of apparent depth. In this article, the term depth refers to the distance between two objects in the visual direction, whereas distance refers to the egocentric distance (i.e., the distance from the observer to the object). 
We can also consider the situation in which the viewing distance is not known, and $d_{0}$ in Equation (1) is a free parameter. In this case, depth-of-field blur can be used to estimate the viewing distance. In Equation (1), $d_{0} / d_{1}$ can be obtained from other perspective cues, such as relative size. This implies that the blur circle is inversely proportional to the focal distance $d_{0}$ (see Equation (1)). Hence, a large blur gradient caused by a small depth of field is consistent with a short viewing distance. Note that if depth-of-field blur is used to estimate viewing distance in this way, it may also be expected to affect apparent size. Thus, a large blur gradient, indicating a close-by viewing distance, would be expected to create a miniaturization effect through scaling of retinal information to estimate object size. Depthof-field blur could thus be used to provide a cue both to the relative depth of objects in the scene and as a way of estimating the egocentric distance to the scene, and thus as a way of scaling relative cues so as to provide absolute depth information. Previous research has shown both effects of depth of field in nonstereo photographs, depending on the availability of other cues to egocentric distance.

Nefs [2012] demonstrated the influence of depth of field on the perceived depth/width relationship in nonstereo photographs of natural scenes. In his study, the photographs contained a central figurine with a flanking figurine at each side. The observers were asked to move the flanking figurines to such a position that the distance between them and the depth to the central figurine were the same. Five levels of depth of field were used by manipulating the camera aperture. He found that perceived depth, relative to perceived width, increased as the depth of field became smaller. In these experiments, the viewing distance was well defined, since the focal plane was always on the computer screen. Thus, the magnification of relative depth with decreasing depth of field found was consistent with the use of depth-of-field blur as a relative depth cue. Held et al. [2012] have also argued that blur might be used as a cue to depth in this way, showing that under some circumstances it can be more reliable than binocular disparity.

Depth-of-field blur can also affect observers' estimates of egocentric distance. For example, Held et al. [2010] asked participants to estimate the distance from a target in the scene to the camera. They found that the reported camera distance was smaller for photographs with larger blur gradients than for photographs with smaller blur gradients. Vishwanath and Blaser [2010] found that perceived egocentric distances were smaller for larger blur gradients. This effect was observed both when observers were estimating pictorial depth in a scene and when they were explicitly asked to judge the distance to the display screen. Although neither of these studies directly assessed the "miniaturization" effects that can occur when the depth of field is reduced (e.g., in tilt-shift photography), both argue that such effects can be attributed to the reduction in apparent egocentric distance.

\subsection{Stereoscopic Viewing}

Binocular disparity provides an important depth cue in stereo displays. Disparity provides precise depth information for points that are close to where one is fixating [Held et al. 2012]. However, using disparity as a cue to depth in current displays can create a conflict between distance information signaled by convergence and accommodation [Hoffman et al. 2008]. This conflict usually causes visual discomfort, particularly when there are frequent, large motor responses of the eyes as a consequence of a large disparity [Lambooij et al. 2009]. Limiting the size of disparity to decrease discomfort has the inevitable consequence that the magnitude of depth that can be signaled will be limited. Previous investigations have suggested that depth-of-field blur may be used to compensate for the loss of perceived depth on 3D displays that is caused by restricting the range of disparities presented [Held et al. 2012; Wang et al. 2011]. Wang et al. [2011] investigated the effect of combining blur and disparity on perceived depth in 3D images. They found that image blur (created using a Gaussian filter) in stereo images contributed to the impression of perceived depth. These results suggest that depth-of-field blur 
may have the same effect on perceived depth in stereo photographs as it does in nonstereo photographs [Nefs 2012].

Wang et al. [2011] used a two-alternative forced-choice task to investigate the apparent depth separation between a foreground target object and a background fronto-parallel plane. The target object was a photograph of a butterfly, and the background was a photograph of a flowerbed. They showed that the perceived depth between the target and the blurred background matched that of a sharp target and a sharp background when the former had a smaller disparity than the latter. Thus, the blur in the background contributed to apparent depth. Our current study assesses whether depth-of-field blur in scenes with complex depth information has similar effects on perceived depth. In the work of Wang et al. [2011], depth information from both disparity and blur were rather simplified. For both, the background and the target, the disparity and blur were constant, indicating a fronto-parallel structure. The only disparity and blur differences present were those between the background and target. This means that neither disparity nor blur depicted a complex depth structure of a type that might be expected in natural scenes. Thus, by using a uniform Gaussian blurring of the entire background, this study did not replicate the depth-of-field blur gradients found in natural images.

In the current work, we investigate the effect of optically created depth of field of different levels on perceived depth in stereo images. There are numerous reasons to believe that the effect of depth of field on perceived depth is not necessarily the same for stereo depth as for nonstereo depth. First, the subjective experience of depth in stereo photographs is qualitatively different from that in nonstereo photographs. In nonstereo photographs, pictorial space does not appear to occupy the same physical space as in stereo images [Rogers 1995]. By contrast, stereo images typically create an experience of stereopsis, which is the sense of a 3D space rather than a flat pictorial space. Importantly, the perceived space appears to be physically embedded in the real world [Vishwanath and Hibbard 2013]. Second, the optical state of the eyes might be more tightly linked to depth of field in stereo photographs than in nonstereo photographs. In the latter case, optical accommodation and binocular convergence typically provide cues to the location of the pictorial surface but not to the objects in the scene. Indeed, when the link between accommodation and convergence is weakened, a greater sense of stereopsis is experienced even when viewing nonstereo images [Vishwanath and Hibbard 2013] and the sense of spatial presence is enhanced [Ling et al. 2013]. This suggests that the effect of depth of field on perceived egocentric distance is likely to be minimal because stereo photographs contain relatively strong depth cues. This means that the depth miniaturization effects discussed by Held et al. [2010] and Vishwanath and Blaser [2010] are unlikely to occur in stereo photographs.

By contrast, if depth-of-field blur provides a relative depth cue that is then scaled by an estimate of egocentric distance, we may expect the degree of apparent depth to increase with decreasing depth of field, as found by Nefs [2012]. At the same time, we acknowledge that the processing of binocular disparity may be impaired with a small depth of field, which may lead to somewhat unpredictable results - that is, the introduction of blur by a small depth of field will tend to reduce the high spatial frequency content in part of the image and thus will reduce the quality of depth information available from disparity. It is well known that the human binocular stereo system is relatively insensitive at low spatial frequencies [Arndt et al. 1995]. This means that an increase in blur, while in itself acting to increase apparent depth, may have the effect of reducing the magnitude of depth perceived from binocular disparity. This loss of binocular disparity may either lead to an increase or a decrease in perceived distance depending on the distances specified by other depth cues.

\subsection{Current Study}

In the current work, we measured the perceived position of a probe in a photograph of a scene while varying the degree of depth of field. Observers were asked to adjust the position of a small rectangle in 
an experimental interface corresponding to the position of the probe in the scene. A reference distance was provided to match the distance in the real world with the distances in the experimental interface. Two experiments were performed, in which the stereoscopic images were presented (1) using a Wheatstone stereoscope and (2) a commercially available 3D television with passive shutter glasses. The Wheatstone stereoscope is a traditional stereo display that can be used to present high-quality stereo stimuli; it is a very tightly controlled presentation method that allows for comparison with previous laboratory research. The $3 \mathrm{D} \mathrm{TV}$, on the other hand, is a new technology that is becoming more common in home entertainment systems and is considered important to investigate the effects of depth of field in a real application. Two display devices were used as follows: (1) to assess how easily potential effects of depth of field could be replicated across different viewing systems, and (2) to allow us to vary the viewing distance. Considering that the 3D TV was much larger than the Wheatstone stereoscope, the viewing distance could also be much larger. According to geometrical considerations and previous research [Gooding et al. 1991; Ritter 1977; Watt et al. 2005], we predicted that the perceived depth on the 3D TV would be larger than on the stereoscope due to the larger viewing distance combined with the same angular disparities in the two cases. Since the stimuli in these two experiments contained another important depth cue - that of height-in-the-field cue [He and Ooi 2000] —we conducted an extra experiment on the 3D TV, using stimuli without this height-in-the-field cue to investigate the influence of other depth cues on the effect of depth of field on perceived depth.

\section{EXPERIMENT}

\subsection{Participants}

Sixty participants, between 21 and 35 years of age, with normal or corrected-to-normal visual acuity (measured with the Freiburg visual acuity test) and normal or better-than-normal stereoacuity (measured with the TNO stereo test), took part in the experiments. All participants were naïve to the hypotheses of the experiments. This research was approved by the Ethic Committees of the Delft University of Technology and was in accordance with the 1969 Declaration of Helsinki.

\subsection{Apparatus}

A Wheatstone stereoscope with two 19" MM904UT Iiyama CRT monitors and front surface silverplated mirrors was used in the first experiment to present the stereo photographs. The path length between the eyes and the screens was $70 \mathrm{~cm}$. The two monitors were calibrated with a ColorMunki spectrophotometer such that their luminance and color responses were identical. The second experiment was a replication of the first, using a TX-P65VT30E Panasonic 65" plasma 3D TV with passive shutter glasses. We used the side-by-side 3D mode as the stereo signal format. All stimuli were presented using a Mac Pro computer running OSX 10.6. An IBM ThinkPad was used as an interface to collect the participants' responses. The experimental interface was presented using MATLAB R2011b.

\subsection{Stimulus}

Stereo photographs were taken with an Olympus E-440 d-SLR camera with an Olympus Zuiko 50-mm macro lens. An example of the stereo photographs is shown in Figure 1. The aperture of the lens could be adjusted from F2 to F22. In our experiments, F2, F3.5, F6.3, F11, and F22 (lens aperture = focal length $/ \mathrm{F}$-value $=25,14.3,7.9,4.5$, and $2.3 \mathrm{~mm}$, respectively) were used to create different depths of field. The visual angle of the camera was $13.2^{\circ}$ (horizontally) $\times 9.9^{\circ}$ (vertically). The size of the stimuli was constrained by the visual angle of the camera and the dimensions of the display systems. The visual angle of the stimuli on the stereoscope and the 3D TV was adjusted to the visual angle of the camera. To keep the visual angle identical for the Wheatstone stereoscope (with a screen diameter of 
Left eye

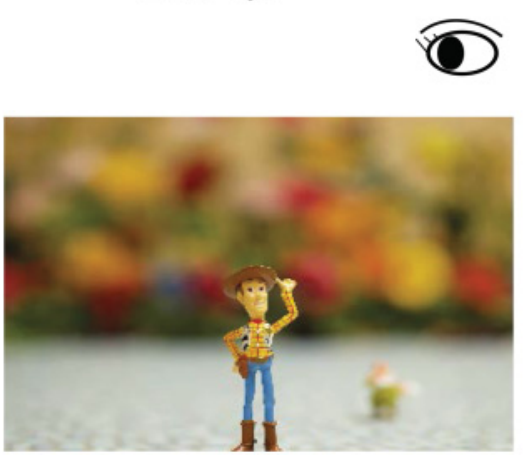

Right eye

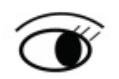

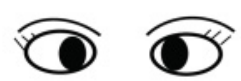

Left eye

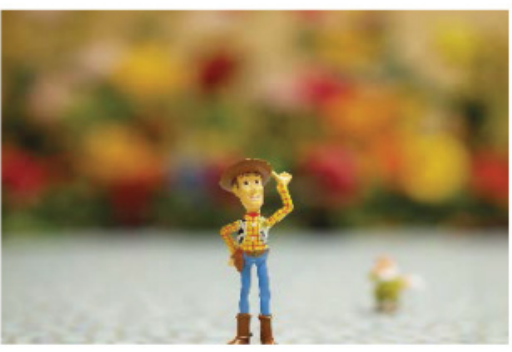

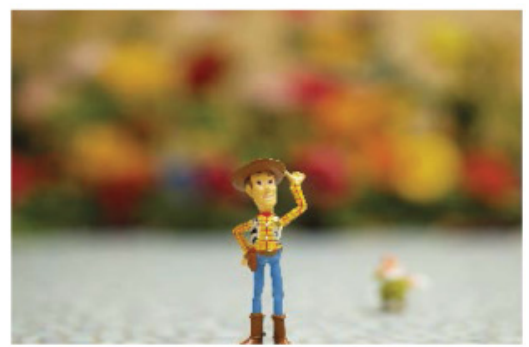

Fig. 1. Examples of stereo photographs. Readers capable of free fusion of stereo photographs may use the left and center photographs for uncrossed free fusion, and the center and right photographs for crossed free fusion.

$19 ")$ and the 3D TV (with a screen diameter of 65"), the observers were seated at the center of projection and the viewing distance was $70 \mathrm{~cm}$ for the stereoscope and $300 \mathrm{~cm}$ for the $3 \mathrm{D}$ TV (accounting for a factor of 4.3 difference in horizontal size). We took stereo photographs of the scene with a stereobase of $6.5 \mathrm{~cm}$ (i.e., averaged horizontal distance between the eyes in adults) for presentation on the Wheatstone stereoscope. For presentation on the 3D TV, the stereobase of the cameras was reduced by the same scaling factor (i.e., 4.3) with which the viewing distance was increased to display the same angular disparity. As such, the stereobase of the cameras became $1.5 \mathrm{~cm}$ for presentation on the 3D TV. In our stimuli, the focus objects were on the same level as the camera and the camera was toed in. As a consequence, incorrect vertical disparities might have been introduced when the stimuli were projected, which might have affected the perception of depth [Banks et al. 2012]. Although the target and flanker objects themselves were close to fixation, and therefore unlikely to provide usable vertical disparity information in themselves, it is important to also consider the fact that vertical disparities in the surround might also have affected the perceived distance of the targets [O'Kane and Hibbard 2007]. We calculated the maximum error of the vertical disparities between the real scene and the images and found it to be relatively small (less than 0.5 arc min). Moreover, since the overall extent of the projected images was considerably less than 20 degrees, vertical disparities were unlikely to be effective [Bradshaw et al. 1996]. Although we cannot rule out any such effects, the vertical disparities present in our stimuli were not affected by the depth of field.

The stereo stimuli used in our experiments consisted of a colorful background containing a broad range of spatial frequencies made up of flowers and leaves, a ground plane, and two objects standing on the ground plane. One of the objects was a reference (Woody from the movie Toy Story) positioned in the center of the scene at zero parallax. The other object was used as the probe, which was positioned at one of the 14 positions behind Woody on the ground plane (Figure 2). When making the stereo photographs, the camera was always aimed at Woody's nose for both the left- and right-half images. The distance between the camera and Woody was $70 \mathrm{~cm}$. Three probes were used, namely a Snow White (SW), a Dwarf (DW), and a Green Pushpin (GP) figurine.

\subsection{Procedure}

We used a between-group design in this experiment. In the experiment, 30 observers participated using the stereoscope, and another 30 observers participated with the 3D TV. The experimental procedure was identical for both groups. Observers were seated in a room with the only direct light coming from the monitors of the Wheatstone stereoscope/3D TV and the IBM ThinkPad. For the Wheatstone 


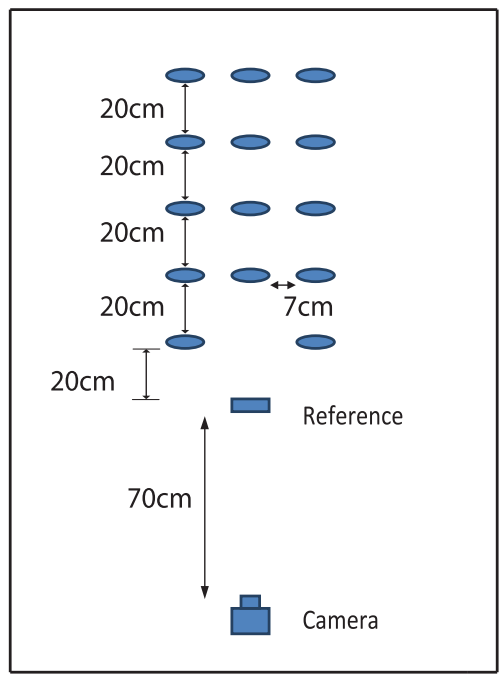

Fig. 2. Layout of 14 locations.

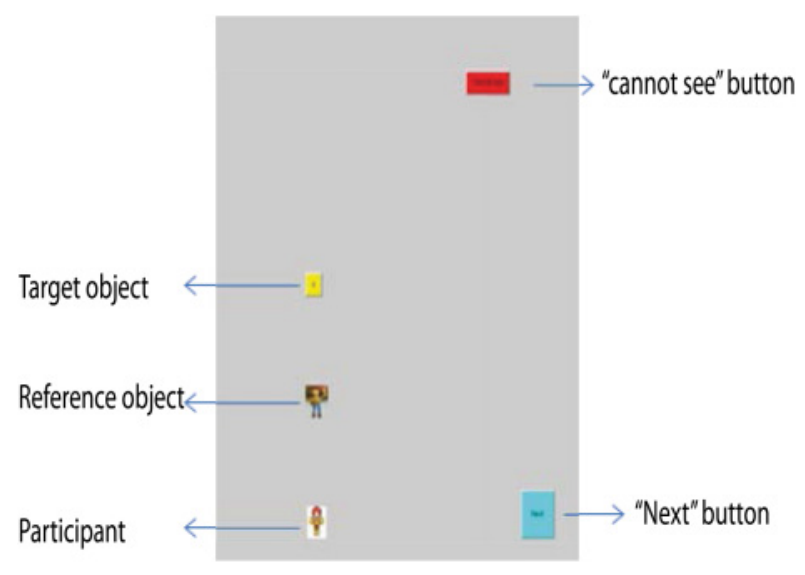

Fig. 3. The experimental interface.

stereoscope, participants used a chin rest in front of the mirrors. For the 3D TV, observers wore shutter glasses, but their head movements were not constrained. Next to the participants was the IBM ThinkPad, on which they could indicate the position of the probe as they perceived it relative to their own sitting position and the reference object. We did not give explicit instructions to observers on how to scale the $Z$ dimension to a setting in the $Y$ dimension other than that the positions of the observer and the reference object were given in the interface. As such, the settings in the $Y$ dimension were assumed to be proportional to the scale in the $Z$ dimension. Figure 3 shows the experimental interface. It contained a small image of a cartoon human and of Woody to represent the positions of participant and reference object, respectively, in the real world. Observers were asked to drag the small yellow square representing the probe into the position where they thought it should be using the distance between the observer and the reference object as a guide. The observers had two degrees of freedom in moving the target object when doing the task. They could move the target object either in the vertical direction to represent the depth or in the horizontal direction to represent the width. They were also free to look at wherever they wanted in the stereo photographs, and the viewing time was unlimited.

When the depth of field was small, it was sometimes difficult to discern the probe in the stereo photographs. Furthermore, the probe was also occluded by the reference object in some positions. This latter situation was more often the case for the 3D TV, as the stereobase of the camera was smaller for the stereo photographs displayed on the 3D TV than on the stereoscope. For this reason, the interface incorporated a "cannot see" button. There were 210 trials in total, including five levels of depth of field, 14 probe positions, and three probes. Each trial was presented only once. The order of the trials was randomized anew for each participant. The experiment took around 30 minutes for each participant.

\section{COMPARISON BETWEEN THE STEREOSCOPE AND THE 3D TV}

Considering that two stereo systems were used in our study, it is necessary to be clear about the differences between the systems. Table I summarizes the parameters for the two setups. Under these circumstances, the horizontal angular disparity between the flanker objects and the focus object was the same when observed through the stereoscope and the 3D TV (e.g., the angular disparity between the first right flanker object and Snow White was 0.279 degrees on both the stereoscope and the 
Table I. Comparison Between the Stereoscope and the 3D TV

\begin{tabular}{|l|l|l|}
\hline & \multicolumn{1}{|c|}{ Stereoscope } & \multicolumn{1}{c|}{ 3D TV } \\
\hline Stimulus-Stereobase & $6.5 \mathrm{~cm}$ & $1.5 \mathrm{~cm}$ \\
\hline Stimulus-Size (on the screen) & $16.2 \mathrm{~cm} \times 12.1 \mathrm{~cm}$ & $69.4 \mathrm{~cm} \times 52 \mathrm{~cm}$ \\
\hline Viewing Distance & $70 \mathrm{~cm}$ & $300 \mathrm{~cm}$ \\
\hline Visual Angle & $13.2^{\circ} \times 9.9^{\circ}$ & $13.2^{\circ} \times 9.9^{\circ}$ \\
\hline
\end{tabular}

3D TV). As shown in Table I, the visual angle subtended by the stimuli was the same on the two displays. Hence, the rendered volume in the visual space was constant on the two displays, which means that not only the size of the stimuli was scaled up but also the stereoscopically presented depth was scaled up proportionally. Thus, the combination of a greater viewing distance with the same angular size of images may be expected to lead to a larger perceived depth on the 3D TV.

\section{RESULTS}

Before proceeding with the main analyses, we calculated how well each probe could be seen at each position. If more than half of the participants indicated that they could not see the probe in a particular position, then that particular condition was defined as on that could not be seen and was excluded from the analysis. In the experimental interface, the vertical/horizontal distance between the yellow square and the reference object was recorded in units of pixels. Given the distance between the participant and reference object, we could calculate the equivalent relationship between perceived depth/width in the experimental interface in pixels and perceived depth/width in the real world in centimeters. Therefore, perceived depth/width refers to the vertical/horizontal distance between the yellow square and the reference object in centimeters in the interface, whereas physical depth/width refers to the distance in depth/width between the probe and the reference figurine in the real world in centimeters.

Figures 4 and 5 show the stereoscopically presented positions (red dots and full lines) and the corresponding perceived positions (blue dots and lines, averaged across 30 participants) of all three probes in the images with five levels of depth of field presented on the stereoscope and on the 3D TV. The black dots represent the stereoscopically presented positions where the probes could not be observed. The stereoscopically presented positions on the stereoscope were the same as the physical positions in the real world. The stereoscopically presented positions on the 3D TV, however, were scaled (with a factor of 4.3) to account for the larger viewing distance, keeping the angular dimensions of the rendered volume in visual space equal for both display systems. Note that the red dots in Figure 5 are not exactly a factor 4.3 more apart than the red dots in Figure 4 because of scaling to fit the graphs on one page. This scaling, however, did not affect the relative positions between dots.

It is obvious from Figures 4 and 5 that the positions of the probes observed through the stereoscope were scaled up in both the horizontal (width) and the vertical (depth) direction, whereas on the 3D TV the perceived positions were scaled up in the horizontal direction only and very close to the stereoscopically presented positions in the vertical direction. To better compare the results on both the stereoscope and the 3D TV in terms of perceived depth, Figure 6 shows the relationship between stereoscopically presented depth and perceived depth. The figure clearly illustrates that the perceived depth on both the stereoscope and the 3D TV increases with increasing stereoscopically presented depth. Additionally, perceived depth is very close to the stereoscopically presented depth on the 3D TV (i.e., the data fall very close to the diagonal of the graph), whereas observers overestimate the presented depth on the stereoscope.

In the following analyses, we use the ratio of perceived depth over physical (presented) depth to describe the effect of depth of field and physical depth on depth perception. Here, physical depth represents the depth between the objects in the real world. Since the perceived depth is inclined to increase 

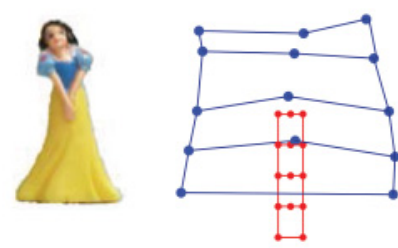

SW

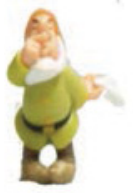

DW
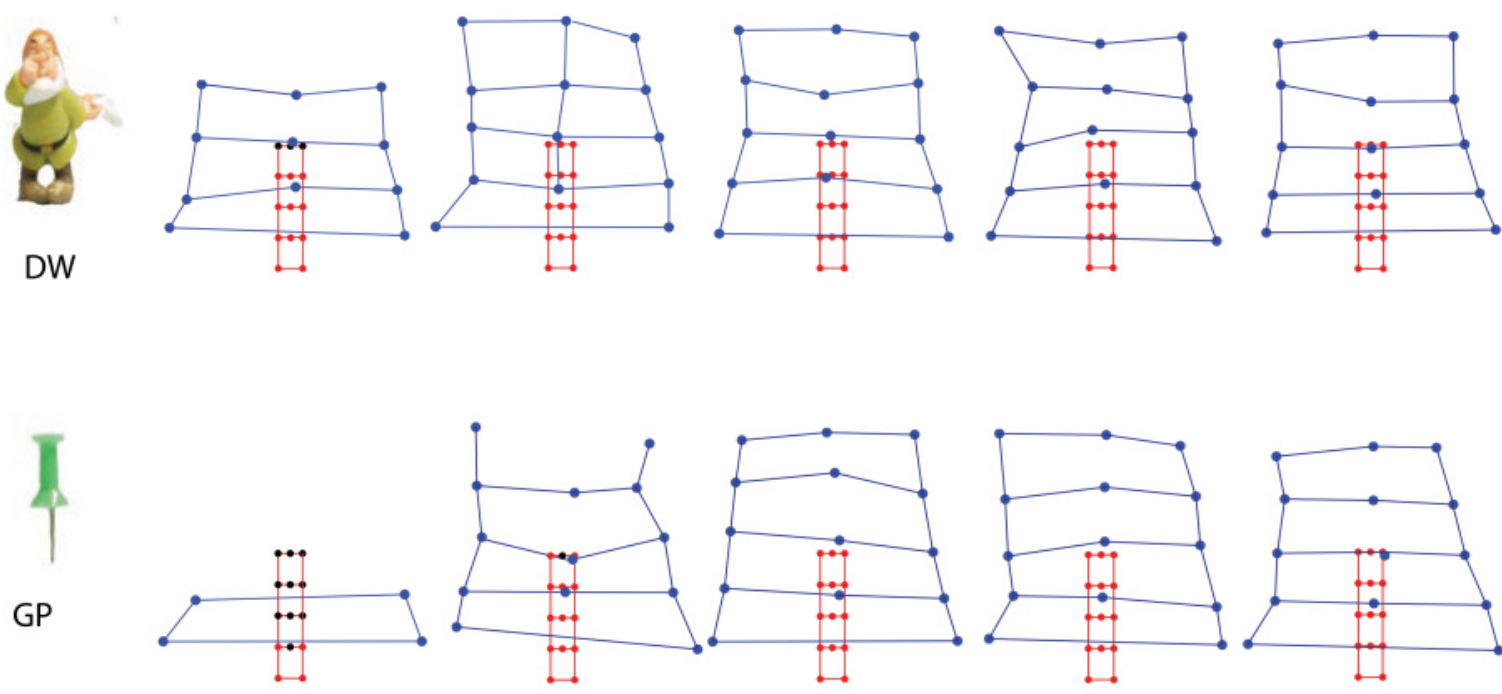

F2
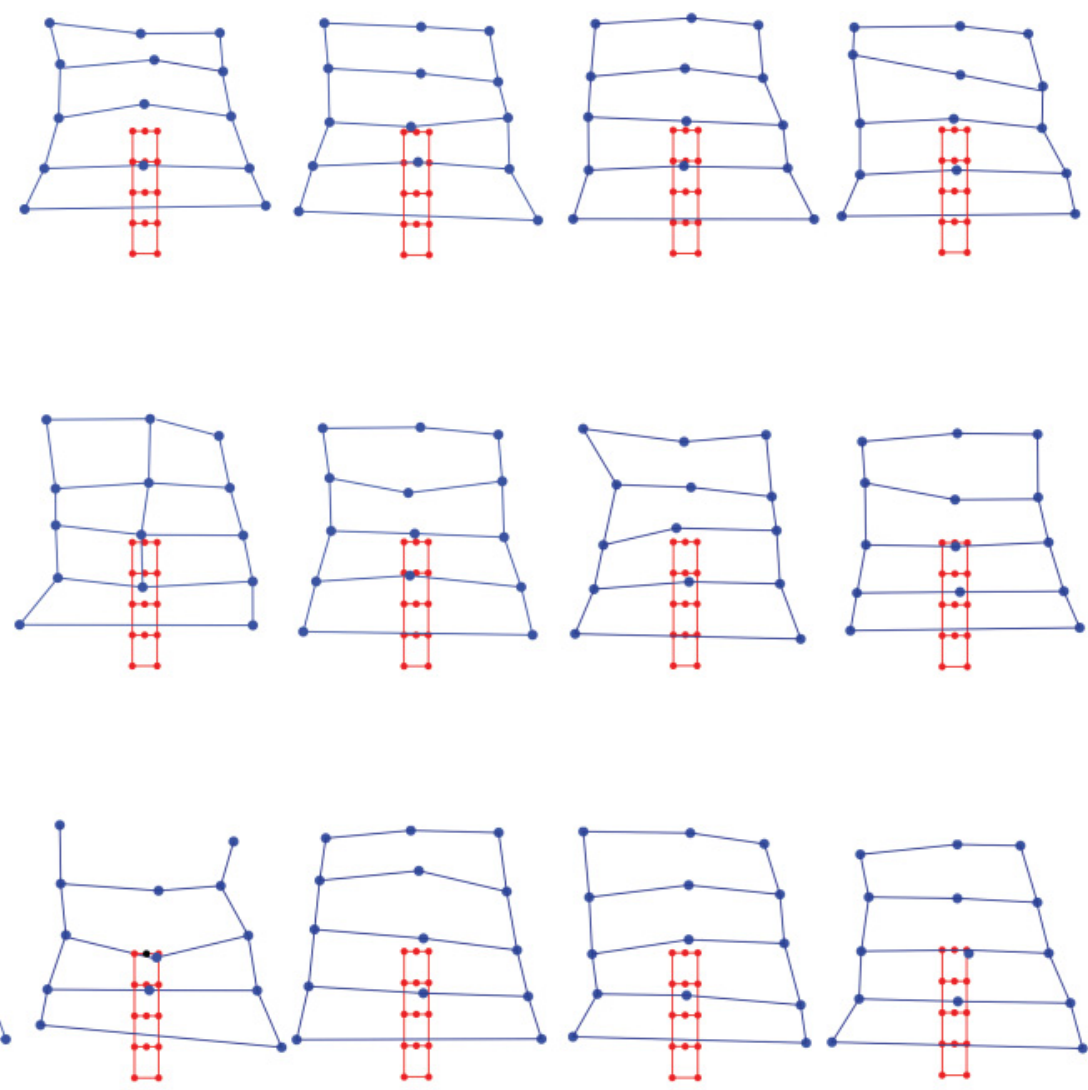

F3.5

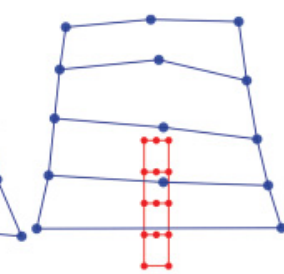

F6.3

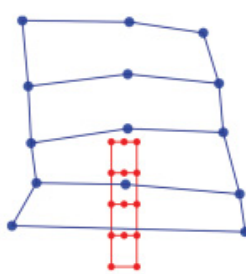

F11

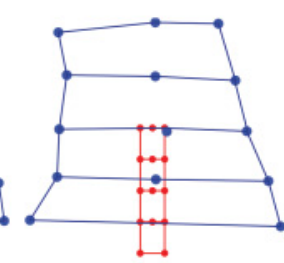

F22

Fig. 4. Layout of the mean perceived positions per figurine on the stereoscope. The red lines indicate the relationship of realworld objects used to make the photographs, and the blue lines indicate the average estimates of observers for the three probes.

with increasing physical depth, it is better to use the ratio of perceived depth over physical depth to describe the effect of depth of field on perceived depth compared with just the value of perceived depth. The ratio of perceived depth/physical depth is averaged over all three probe conditions as well as across positions on the same row, excluding the missing data. In the rest of the article, we refer to this average when using the ratio of perceived depth/physical depth.

For stimuli presented on the stereoscope, the ratio of perceived depth/physical depth across all conditions for all participants ranged from 0.56 to 5.53. Figure 7(a) shows that for stimuli viewed on the stereoscope, perceived depth decreased with increasing depth of field from F3.5 to F11, whereas perceived depth increased from F2 to F3.5. Figure 7(b) illustrates that the ratio of perceived depth/physical depth decreased with increasing physical depth from $20 \mathrm{~cm}$ to $100 \mathrm{~cm}$. Figure $7(\mathrm{c})$ demonstrates that the ratio of perceived depth/physical depth decreased with increasing physical depth for all the five levels of depth of field. We performed a 5 (Depth of field) $\times 5$ (Physical depth) repeated measures ANOVA and found main effects of Depth of field $(F(4,116)=4.44, p<.002)$ and Physical depth $(F(4,116)=39.35, p<.001)$. A significant interaction between Depth of field and Physical depth was also found $(F(16,464)=2.98, p<.001)$. The results of post hoc pairwise comparison $t$-tests are also 


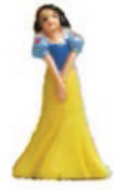

SW
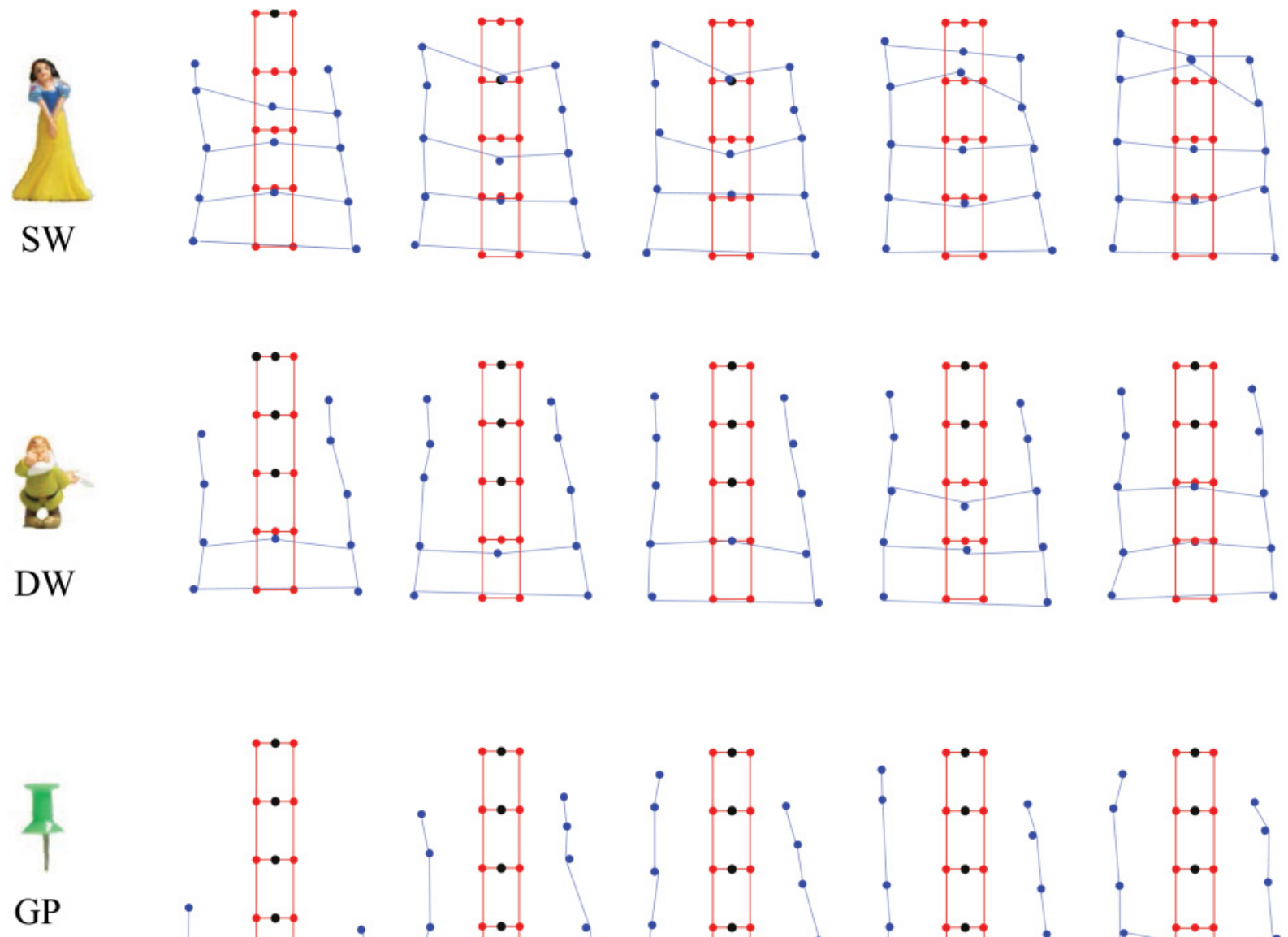

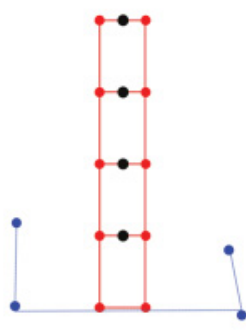

F2

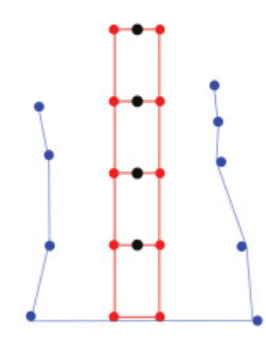

F3.5

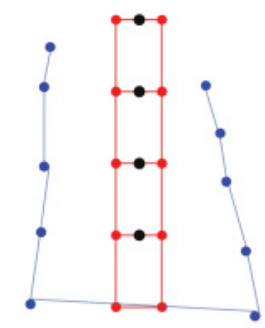

F6.3

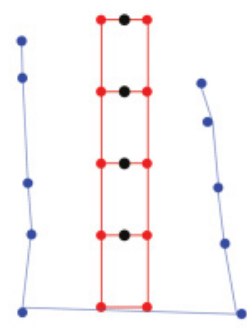

F11

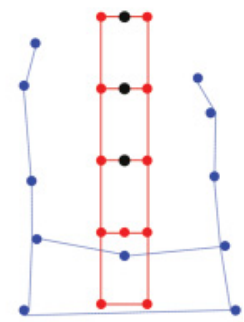

F22

Fig. 5. Layout of the mean perceived positions per figurine on the 3D TV. The red lines indicate the relationship of real-world objects used to make the photographs, and the blue lines indicate the average estimates of observers for the three probes.

shown in Figure 7, with the asterisk indicating when there was a significant difference between a pair of depth of field levels.

For stimuli presented on the 3D TV, the mean ratio of perceived depth/physical depth across all conditions ranged from 1.74 to 7.74. The data are summarized in Figure 8. On visual inspection, Figure 8(a) shows that perceived depth at depth of field F2 was smaller than at all other depths of field, and that perceived depth was not affected by depths of field larger than F3.5. Figure 8(b) illustrates that perceived depth, relative to width, decreased with increasing physical depth. Finally, Figure 8(c) shows that the ratio of perceived depth/physical depth generally decreased with increasing physical depth. We performed a 5 (Depth of field) $\times 5$ (Physical depth) repeated measures ANOVA and found main effects of Depth of field $(F(4,116)=10.87, p<.001)$ and Physical depth $(F(4,116)=8.77$, $p<.001$ ). A significant interaction between the Depth of field and Physical depth was also found 


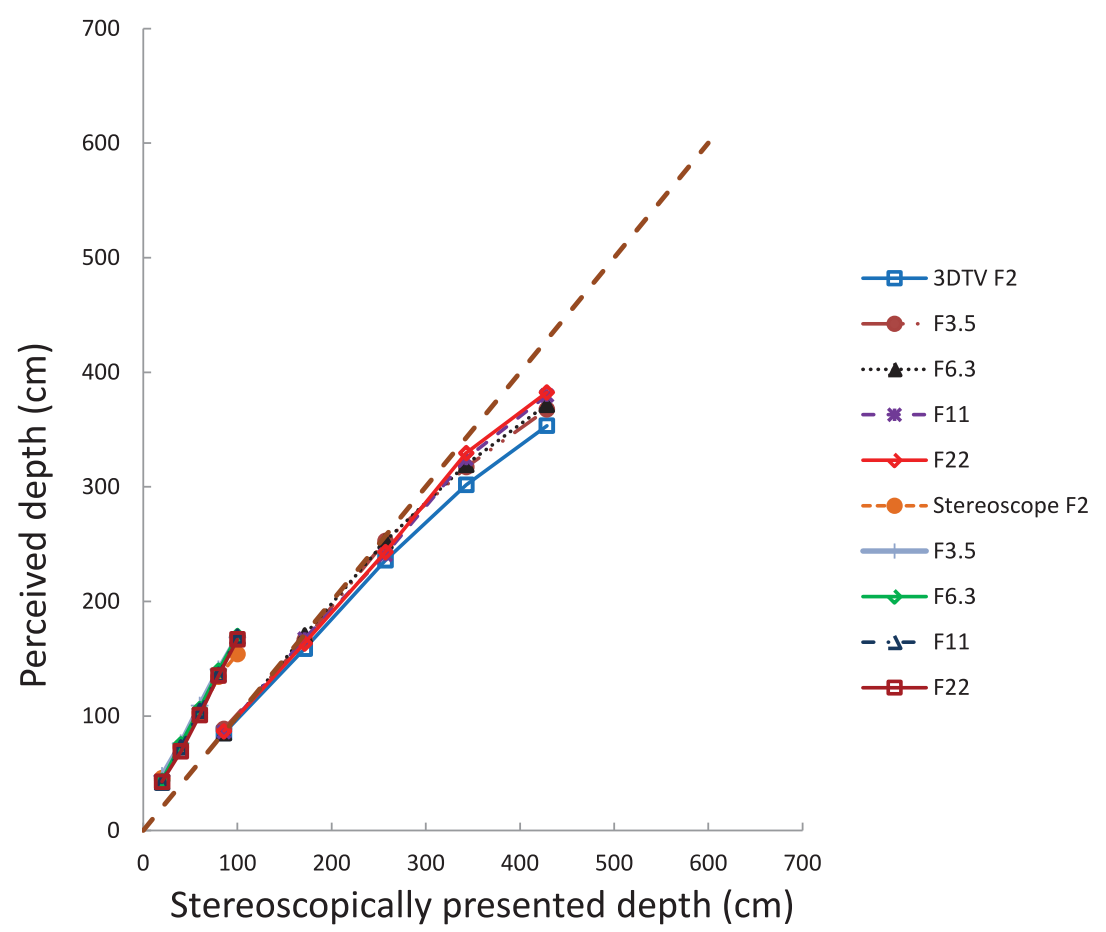

Fig. 6. The relationship between perceived depth and stereoscopically presented depth for all depths of field on both the stereoscope and the 3D TV. Relative depth is the stereoscopically indicated distance in depth between the focus object and the flanker. Perceived depth is the average estimated depth made by all observers.

$(F(16,464)=2.55, p<.001)$. Figure 8 also shows the results of post hoc pairwise comparison $t$-tests, with the asterisk representing that there was significant difference between the each of the pairs of depth of field values.

\subsection{Additional Control Experiment: Removing the Height-in-the-Field Cue in the Stimuli on the 3D TV}

The results reported previously showed that depth of field did not influence perceived depth in the range between F3.5 and F22. When preparing the stimuli, the cameras were focused and converged on the central figurine from a greater height above the ground surface than the nose. As a consequence, the distance from the probe to Woody could to some degree be estimated from its height in the visual field (Figure 9(a)). Literature shows that the surface ground can help observers make accurate distance judgments in stereoscopic viewing conditions [He and Ooi 2000]. Therefore, it is possible that the effect of depth of field on perceived depth was weakened in our experiment because of the height-in-the-field cue or surface ground information. Consequently, we hypothesized that depth of field may have a stronger influence on perceived depth in stereoscopic photographs without a height-in-the-field cue (e.g., Figure 9(b)) than in photographs with a height cue (e.g., see Figure 9(a)).

To investigate this hypothesis, we created some new stimuli, as shown in Figure 9(b), with the influence of the surface ground occluded. The experimental setup was the same as for the experiment on the 3D TV described in Sections 2.2 and 2.3. In this additional control experiment, we only used the Snow White probe, but still for the 14 positions and five different levels of depth-of-field blur. To remove the height cue, we set the camera at the height of the ground surface, taking care that Woody, Snow White, and the background were at the same height in the photograph (see Figure 9(b)). To make 

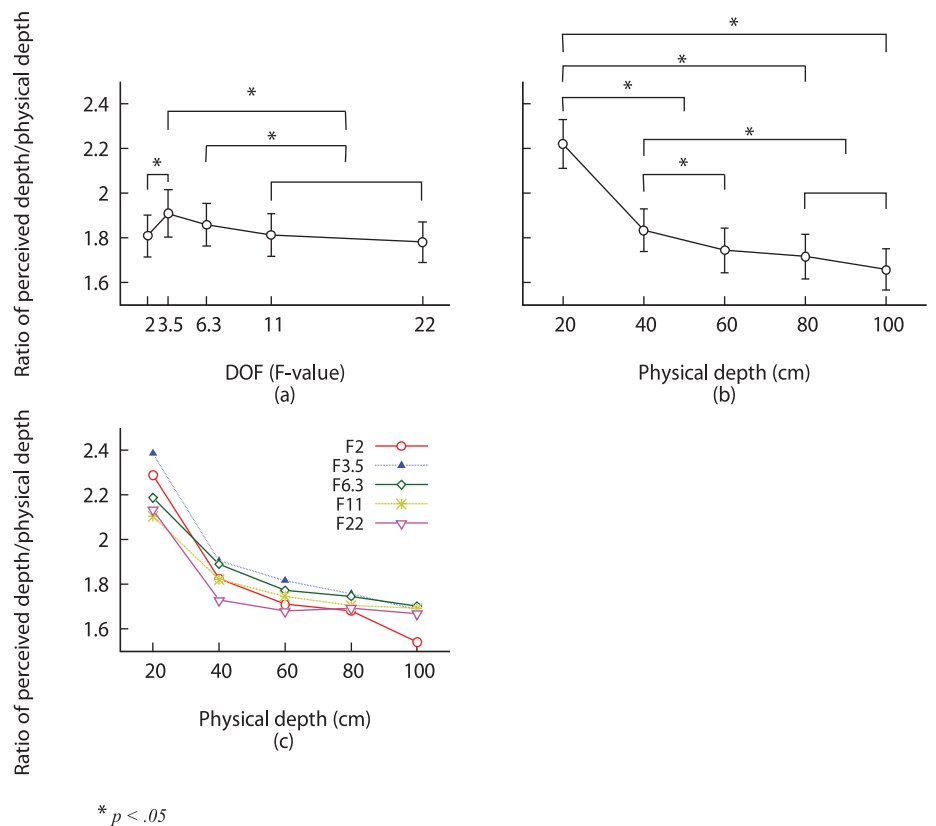

Fig. 7. Mean values of ratio of perceived depth/physical depth as measured on the stereoscope based on a 5 (depth of field) $\times 5$ (physical depth) experimental design. Error bars show \pm 1 standard error of the mean. The ratio of perceived depth/physical depth as a function of (a) depth of field collapsed across two probes and four physical depths, (b) physical depth collapsed across two probes and five depths of field, and (c) physical depth collapsed across probes, but for the various depths of field separately.

the stimuli consistent with the previous ones, we cropped all of the images so that the differences in height in the visual field between objects were removed. Participating in the experiment were 17 naïve observers with a mean age of 27.6 years $(\mathrm{SD}=4.5)$. The experimental procedure was again the same as described for the main experiment on the $3 \mathrm{D}$ TV.

Figure 10 shows that the ratio of perceived depth/physical depth decreases with increasing depth of field. This means that objects in the image with more blur are perceived as farther away than the same objects in images with less blur, showing that depth-of-field blur can act as a depth cue in stereoscopic photographs. We performed a 5 (Depth of field) $\times 5$ (Physical depth) repeated measures ANOVA and found a significant effect of Depth of field $(F(4,64)=3.76, p<.008)$ and of Physical depth $(F(4,64)=36.49, p<.001)$ on perceived depth. The effect of depth of field from F2 to F3.5 in this experiment is opposite to what we found in the stimuli with the height cue in the visual field. The effect of depth of field from F3.5 to F22 turns out to be significant and consistent with what we found on the stereoscope for photographs with a height-in-the-field cue in the visual field.

\section{DISCUSSION}

We have shown that depth of field may affect perceived depth in stereo photographs using a stereoscope at a closer viewing distance or a 3D TV at a larger viewing distance. First, for both systems, the individual range of the ratio of perceived depth/physical depth for all participants under all conditions was large (i.e., from 0.56 to 5.53 on the stereoscope and from 1.74 to 7.74 on the 3D TV). Second, perceived depth increased with increasing depth of field from F2 to F3.5 on both systems when the height-in-the-field cue in the images was congruent with the depth of field. Third, perceived depth, as a proportion of physical depth, decreased with increasing physical depth on both systems. 


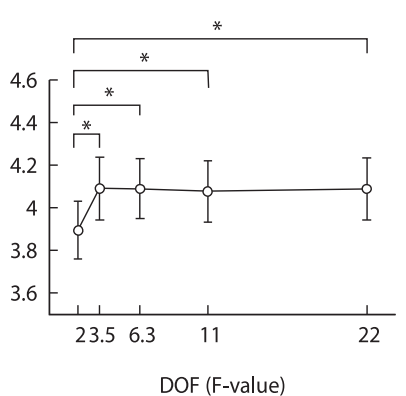

(a)

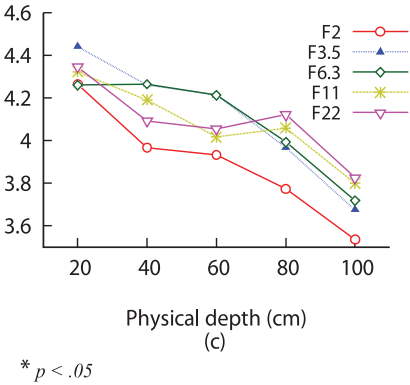

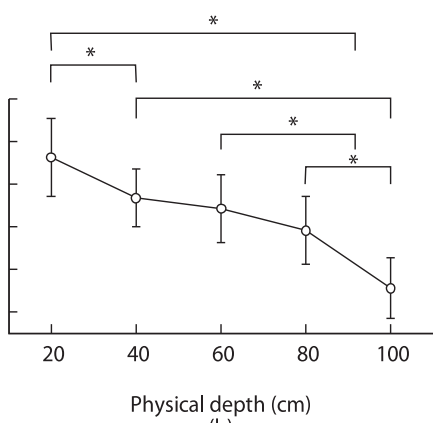

(b)

Fig. 8. Mean values of ratio of perceived depth/physical depth as measured on the 3D TV based on a 5 (Depth of field) $\times 5$ (Physical depth) experimental design. Error bars show \pm 1 standard error of the mean. Ratio of perceived depth/physical depth as a function of (a) depth of field collapsed across all probes and physical depths, (b) physical depth collapsed across all probes and depths of field, and (c) physical depth collapsed across depth of field, but for the two probes separately.

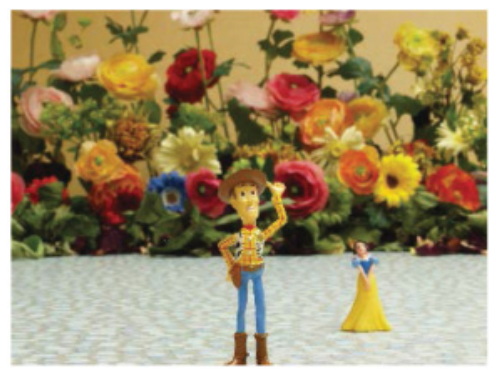

(a)

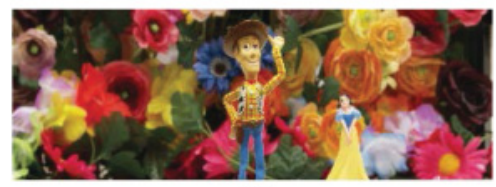

(b)

Fig. 9. (a) An example stimulus used in the main experiment containing the height-in-the-field cue. (b) The same stimulus used in the control experiment but now without a height-in-the-field cue. 


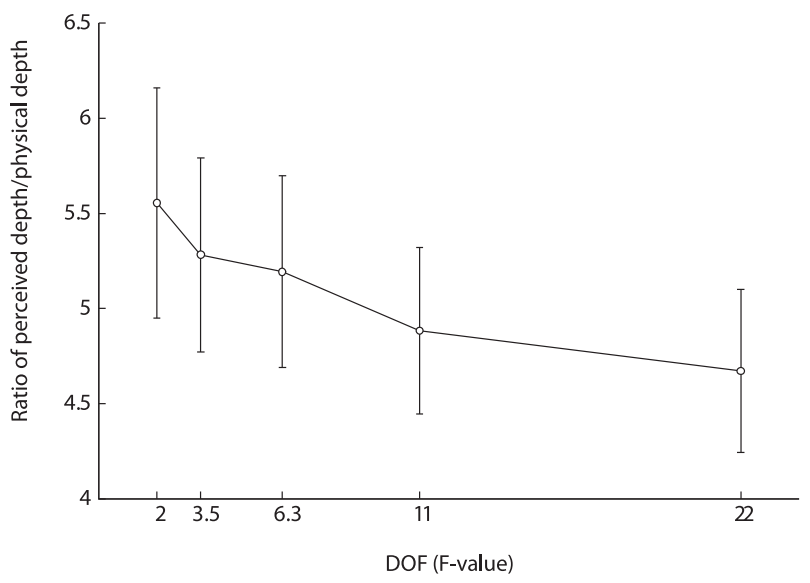

Fig. 10. Ratio of perceived depth/physical depth as a function of depth of field in the stimuli without height cue collapsed across physical depths.

We predicted that if blur is used as a depth cue, then decreasing the depth of field would result in an increase in perceived depth. In the main experiments, our results were partly consistent with this hypothesis. Perceived depth decreased as a function of depth of field beyond F3.5 on the stereoscope. For depth of field between F2 and F3.5, however, our results contradicted our hypothesis. The ratio of perceived depth/physical at the smallest depth of field F2 was less than that at F3.5. The most likely reason for this is that at the smallest depth of field, the degree of blur introduced reduced the quality of disparity information provided [Costa et al. 2010]. As such, a smaller depth of field may have resulted in less perceived depth under stereoscopic viewing conditions. Another possible reason is that the very small depth of field introduced so much blur that the visibility of details of the probes were firmly reduced. The third reason may be that the distance to the scene was more tightly constrained in the case of stereo viewing than in the case of nonstereo viewing (e.g., Held et al. [2010]) or viewing through an aperture (e.g., Vishwanath and Blaser [2010]), which caused the divergence in results between stereo and nonstereo photographs. Changes in depth of field may thus be expected to have less of an effect on perceived egocentric distance under stereoscopic viewing.

On the 3D TV, no significant difference in perceived depth was found with increasing depth of field in the range from F3.5 to F22 for photographs with a height-in-the-field cue. However, an additional control experiment showed that perceived depth decreased with increasing depth of field significantly if the height-in-the-field cue was removed from the stimuli. This suggests that the effect of depth of field on perceived depth on the 3D TV may be greatly weakened by other depth cues. But, the results of the control experiment also demonstrate that depth-of-field blur can indeed act as a depth cue even under binocular viewing conditions. When height-in-the-field depth cues are absent, the amount of depth indicated by our observers increased monotonically with increases in depth-of-field blur. As such, these results are consistent with the suggestion of Held et al. [2012] that blur provides a quantitative depth cue under binocular viewing. This study was criticized by Vishwanath [2012] in part because it did not directly demonstrate the effect of blur on the magnitude of perceived depth. For nonstereoscopic photographs, Nefs [2012] provided direct evidence for the effect of depth-of-field blur on perceived depth, consistent with the use of blur as a depth cue. Here, we show that these effects may also occur in stereoscopic viewing.

Physical depth was found to significantly affect perceived depth in stereo photographs in our experiments. We found a clear overestimation of depth between the focus object and the probe compared 
to the physical depth on both the stereoscope and the 3D TV, as shown in Figures 7(c) and 8(c). The reasons for this overestimation are different for the two display systems. For the stereoscope, the stereobase of the cameras recording the images was similar to the human adult interocular separation, and the viewing distance and projected size of the images matched those used for the cameras. Binocular depth cues thus indicated the correct depth in this case, so the biases observed represent a genuine overestimation of depth. It is known that perceived depth is overestimated in this way for objects viewed at close distances, both for stereoscopically viewed [Johnston 1991; Scarfe and Hibbard 2006, 2011] and natural objects [Loomis et al. 1998; Watt et al. 2005]. The overestimation data from some previous studies are partly consistent with our results, indicating that perceived distance was overestimated compared to the physical distance [Elliott 1987; Loomis et al. 1998]. The overestimation of depth in the case of 3D TV may be related to the 4.3 upscaling factor of the image size, viewing distance, and rendered depth (as explained in Section 2.3). As the ration of perceived depth/physical depth is close to or slightly smaller than 4.3 in Figure 8, it seems that our participants perceived depth very close to the rendered depth on the 3D TV. The present study also showed that observers overestimated depth less with increasing physical depth. This latter finding is consistent with results reported by Sauer [2001] and confirms the conclusion of Gilinsky [1951] that perceived distance is a nonlinear function of physical distance. As shown in Figure 6, we found that the perceived depth is farther away from the stereoscopically presented depth at larger depth on both the stereoscope and the 3D TV. This confirms the results of a previous study that the effective range of stereopsis as a depth cue attenuates with distance [Held et al. 2012]. With increasing physical distance from the observer, the just-discriminable depth threshold also increases [Cutting and Vishton 1995], implying that observers are less certain about differences in depth. As such, the observers' mapping of perceived depth to physical depth could vary more with increasing distance to the objects [Ernst and Banks 2002].

Interestingly, there was a significant interaction between depth of field and physical depth on both the Wheatstone stereoscope and the 3D TV; the effect of depth of field on perceived depth was larger at a smaller physical depth. The reason could be that the human eye perceives depth of field cues most effectively within a certain depth range [Kaufman 1974]. Wang et al. [2011], however, reported a different result, namely that the effect of blur on perceived depth was smaller when the relative distance between a foreground object and the background was closer. The crucial difference between their work and ours was that the relative distance in their study ranged from $0 \mathrm{~cm}$ to $30 \mathrm{~cm}$, whereas in our experiment it varied from $20 \mathrm{~cm}$ to $80 \mathrm{~cm}$. This may be explained by the different importance of disparity and blur in different ranges [Held et al. 2012]. Alternatively, it could be that the differences resulted from the differences between the Gaussian blurring implemented by Wang et al. [2011] and the optical depth of field effects in the current study.

When comparing the results of the two experiments, we found not only many similarities but also an interesting difference. Perceived depth was found to decrease with increasing depth of field from F3.5 to F11 on the stereoscope, whereas no effect of depth of field from F3.5 to F22 was found on the 3D TV when a height-in-the-field cue was present in the images. In other words, it seemed that the effect of depth of field was smaller for the 3D TV than for the stereoscope. There are obviously a number of reasons why the results on the stereoscope and the 3D TV might have been different. Differences in viewing distance, stereobase of the camera, and screen resolution might have directly or indirectly impacted the effect of depth of field on perceived depth. More interestingly, however, is the observation that the impact of depth-of-field blur on perceived depth increased on the 3D TV once the height-in-thefield cue was removed from the stimuli. So, especially on the 3D TV, more than on the stereoscope, the effect of depth of field on perceived depth was weakened by the height-in-the-field cue. The latter may be a direct consequence of the difference in viewing distance between both display systems. At larger 
(viewing) distance, depth-of-field blur is less impactful and perceived depth may become dominated by other pictorial cues, such as the height-in-the-field cue.

Wang et al. [2011] made the point that depth-of-field blur could be used to supplement the subjective impression of depth in stereoscopically presented images. This means that a smaller disparity-defined depth cue would be needed to create the same perception of depth when the depth-of-field blur cue was used as well. As such, depth-of-field blur could indirectly (i.e., via smaller disparity values) reduce the level of accommodation-vergence conflict and yet preserve the subjective impression of depth in the scene. However, given the influence of the height-in-the-field cue on depth-of-field effects in certain viewing conditions, it may be that also other combinations of nonstereoscopic or pictorial cues together with depth of field are useful to reduce the required disparity for a given perceived depth and thus are useful to reduce visual discomfort. For example, it has been suggested that depth of field in combination with shading in a Bayesian framework allows optimal reconstruction of depth relationships in a scene [Li et al. 2013]. An essential condition for the resulting reduction in visual discomfort, however, is that the additional cues and depth of field do not introduce discomfort on their own. For example, inappropriately simulated depth of field may be different from that introduced by human optics and thus lead to unstable perceived depth and possibly discomfort. Vinnikov and Allison [2014] found that the viewing comfort was reduced when depth of field was added as a depth cue. However, it has been shown that depth of field itself does not cause discomfort, even when conflicts with binocular depth cues are large [O'Hare et al. 2013]. Altogether, our results indicate that depth-of-field blurring is a promising technique for reducing stereoscopically induced discomfort while maintaining subjective impressions of depth. Nonetheless, future work is needed to determine exactly how disparity, depth-of-field blur, and other pictorial cues need to be combined to provide the most comfortable depth impression in natural images displayed on stereoscopic screens.

\section{CONCLUSION}

In summary, the current study confirms the importance of depth of field on depth perception in stereo photographs. Stereo depth of field is different from nonstereo depth of field: in nonstereo images perceived depth monotonically decreases with increasing depth of field, whereas in stereo images perceived depth may increase with increasing depth of field, especially at small depth of field and short viewing distances. The latter may be a consequence of the high degree of perceived blur in these images. We may conclude that if depth of field is not very small (i.e., > F3.5), it may be used as a depth cue in stereo photographs: a decrease in depth of field creates an increase in perceived depth.

\section{REFERENCES}

P. Arndt, H. Mallot, and H. H. Bülthoff. 1995. Human stereovision without localized image features. Biological Cybernetics 72 , 279-293.

M. S. Banks, J. C. Read, R. S. Allison, and S. J. Watt. 2012. Stereoscopy and the human visual system. SMPTE Motion Imaging Journal 121, 24-43.

Y. Baveye, F. Urban, and C. Chamaret. 2012. Image and video saliency models improvement by blur identification. In Computer Vision and Graphics. Lecture Notes in Computer Science, Vol. 7594. Springer, 280-287.

M. F. Bradshaw, A. Glennerster, and B. J. Rogers. 1996. The effect of display size on disparity scaling from differential perspective and vergence cues. Vision Research 36, 1255-1264.

M. F. Costa, S. M. Moreira, R. D. Hamer, and D. F. Ventura. 2010. Effects of age and optical blur on real depth stereoacuity. Ophthalmic and Physiological Optics 30, 660-666.

J. E. Cutting and P. M. Vishton. 1995. Perceiving layout and knowing distances: The integration, relative potency, and contextual use of different information about depth. In Perception of Space and Motion, W. Epstein and S. Rogers (Eds.). Academic Press, San Diego, CA, 69-117.

R. Datta, D. Joshi, J. Li, and J. Z. Wang. 2006. Studying aesthetics in photographic images using a computational approach. In Computer Vision-ECCV 2006. Lecture Notes in Computer Science, Vol. 3953. Springer, 288-301. 
D. Elliott. 1987. The influence of walking speed and prior practice on locomotor distance estimation. Journal of Motor Behavior $19,476-485$.

M. O. Ernst and M. S. Banks. 2002. Humans integrate visual and haptic information in a statistically optimal fashion. Nature 415, 429-433.

A. S. Gilinsky. 1951. Perceived size and distance in visual space. Psychological Review 58, 6, 460.

L. Gooding, M. E. Miller, J. Moore, and S.-H. Kim. 1991. Effect of viewing distance and disparity on perceived depth. In Electronic Imaging 91. San Jose, CA, 259-266.

J. R. Hamerly and C. A. Dvorak. 1981. Detection and discrimination of blur in edges and lines. Journal of the Optical Society of America 71, 4,448-452.

Z. J. He and T. L. Ooi. 2000. Perceiving binocular depth with reference to a common surface. Perception 29, 1313-1334.

R. T. Held, E. A. Cooper, and M. S. Banks. 2012. Blur and disparity are complementary cues to depth. Current Biology 22, 5, 426-431.

R. T. Held, E. A. Cooper, J. F. O’Brien, and M. S. Banks. 2010. Using blur to affect perceived distance and size. ACM Transactions on Graphics 29, 2, 1-16.

S. Hillaire, A. Lecuyer, R. Cozot, and G. Casiez. 2008. Depth-of-field blur effects for first-person navigation in virtual environments. IEEE Computer Graphics and Applications 28, 6, 47-55.

D. M. Hoffman, A. R. Girshick, K. Akeley, and M. S. Banks. 2008. Vergence-accommodation conflicts hinder visual performance and cause visual fatigue. Journal of Vision 8, 3, 33.

G. S. Hubona, P. N. Wheeler, G. W. Shirah, and M. Brandt. 1999. The relative contributions of stereo, lighting, and background scenes in promoting 3D depth visualization. ACM Transactions on Computer-Human Interaction 6, 3, 214-242.

E. B. Johnston. 1991. Systematic distortions of shape from stereopsis. Vision Research 31(7-8), 1351-1360.

L. Kaufman. 1974. Sight and Mind: An Introduction to Visual Perception. Oxford University Press.

R. A. Khan, H. Konik, and E. Dinet. 2010. Enhanced image saliency model based on blur identification. In Proceedings of the 25th International Conference of Image and Vision Computing New Zealand (IVCNZ). 1-7.

M. Lambooij, F. Marten, I. Heynderickx, and W. Ijsselsteijn. 2009. Visual discomfort and visual fatigue in stereoscopic displays: A review. Journal of Imaging Science and Technology 53, 3, 1-14.

C. Li, S. Su, Y. Matsushita, K. Zhou, and S. Lin. 2013. Bayesian depth-from-defocus with shading constraints. In Proceedings of the IEEE Conference on Computer Vision and Pattern Recognition. 217-224.

Y. Ling, H. T. Nefs, W. P. Brinkman, C. Qu, and I. Heynderickx. 2013. The relationship between individual characteristics and experienced presence. Computers in Human Behavior 29, 4, 1519-1530.

J. M. Loomis, R. L. Klatzky, J. W. Philbeck, and R. G. Golledge. 1998. Assessing auditory distance perception using perceptually directed action. Perception and Psychophysics 60, 6, 966-980.

G. Mather. 1996. Image blur as a pictorial depth cue. Proceedings of the Royal Society of London. Series B: Biological Sciences $263,1367,169$.

G. Mather and D. R. Smith. 2000. Depth cue integration: Stereopsis and image blur. Vision Research 40, 25, 3501-3506.

H. T. Nefs. 2012. Depth of field affects perceived depth-width ratios in photographs of natural scenes. Seeing and Perceiving 25 , 6, 577-595.

L. O'Hare, T. Zhang, H. T. Nefs, and P. B. Hibbard. 2013. Visual discomfort and depth-of-field. i-Perception 4, 3, $156-169$.

L. M. O'Kane and P. B. Hibbard. 2007. Vertical disparity affects shape and size judgments across surfaces separated in depth. Perception 36, 696-702.

A. P. Pentland. 1987. A new sense for depth of field. IEEE Transactions on Pattern Analysis and Machine Intelligence 9, 4, 523-531.

M. Ritter. 1977. Effect of disparity and viewing distance on perceived depth. Perception and Psychophysics 22, 4, $400-407$.

S. Rogers. 1995. Perceiving pictorial space. In Perception of Space and Motion, W. Epstein and S. Rogers (Eds.). Academic Press, San Diego, CA, 119-163.

C. W. Sauer. 2001. Perceived depth of 3-D objects in 3-D scenes. Perception 30, 6, 681-692.

P. Scarfe and P. Hibbard. 2006. Disparity-defined objects moving in depth do not elicit three-dimensional shape constancy. Vision Research 46, 10, 1599-1610.

P. Scarfe and P. Hibbard. 2011. Statistically optimal integration of biased sensory estimates. Journal of Vision 11, 7, 12.

T. Shibata, J. Kim, D. M. Hoffman, and M. S. Banks. 2011. The zone of comfort: Predicting visual discomfort with stereo displays. Journal of Vision 11, 8, 11.

M. Vinnikov and R. S. Allison. 2014. Gaze-contingent depth of field in realistic scenes: The user experience. In Proceedings of the Symposium on Eye Tracking Research and Applications (ETRA'14). 119-126. 
D. Vishwanath. 2012. The utility of defocus blur in binocular depth perception. i-Perception 3, 541.

D. Vishwanath and E. Blaser. 2010. Retinal blur and the perception of egocentric distance. Journal of Vision 10, 10, 26.

D. Vishwanath and P. B. Hibbard. 2013. Seeing in 3-D with just one eye stereopsis without binocular vision. Psychological Science 24, 1673-1685.

J. Wang, M. Barkowsky, V. Ricordel, and P. Le Callet. 2011. Quantifying how the combination of blur and disparity affects the perceived depth. In Proceedings of SPIE, Vol. 7865. Human Vision and Electronic Imaging XVI, 78650K-78650K-10.

S. J. Watt, K. Akeley, M. O. Ernst, and M. S. Banks. 2005. Focus cues affect perceived depth. Journal of Vision 5, 10, 834-862.

Received November 2013; revised May 2014; accepted August 2014 